\title{
Diabetes mellitus and risk of prostate cancer in the European Prospective Investigation into Cancer and Nutrition
}

Konstantinos K. Tsilidis ${ }^{1,2}$, Naomi E. Allen ${ }^{3}$, Paul N. Appleby ${ }^{1}$, Sabine Rohrmann ${ }^{4}$, Ute Nöthlings ${ }^{5}$, Larraitz Arriola ${ }^{6,7}$, Marc J. Gunter ${ }^{8}$, Veronique Chajes $^{9}$, Sabina Rinaldi ${ }^{9}$, Isabelle Romieu ${ }^{9}$, Neil Murphy ${ }^{8}$, Elio Riboli ${ }^{8}$, Ioanna Tzoulaki ${ }^{2,8}$, Rudolf Kaaks $^{10}$, Annekatrin Lukanova ${ }^{10,11}$, Heiner Boeing ${ }^{12}$, Tobias Pischon ${ }^{12,13}$, Christina C. Dahm ${ }^{14}$, Kim Overvad ${ }^{14,15}$, J. Ramón Quirós $^{16}$, Ana Fonseca-Nunes ${ }^{17}$, Esther Molina-Montes ${ }^{6,18,19}$, Diana Gavrila Chervase ${ }^{6,20}$, Eva Ardanaz ${ }^{6,21}$, Kay T. Khaw ${ }^{22}$, Nick J. Wareham ${ }^{23}$, Nina Roswall ${ }^{24}$, Anne Tjønneland ${ }^{24}$, Pagona Lagiou ${ }^{25,26,27}$, Dimitrios Trichopoulos ${ }^{26,27,28}$, Antonia Trichopoulou ${ }^{25,28}$, Domenico Palli ${ }^{29}$, Valeria Pala ${ }^{30}$, Rosario Tumino ${ }^{31}$, Paolo Vineis ${ }^{8,32}$, H. B(as) Bueno-de-Mesquita ${ }^{8,33,34}$, Johan Malm ${ }^{35}$, Marju Orho-Melander ${ }^{36}$, Mattias Johansson ${ }^{9,37}$, Pär Stattin ${ }^{38}$, Ruth C. Travis ${ }^{1}$ and Timothy J. Key ${ }^{1}$

${ }^{1}$ Cancer Epidemiology Unit, University of Oxford, Oxford, United Kingdom

${ }^{2}$ Department of Hygiene and Epidemiology, University of Ioannina School of Medicine, Ioannina, Greece

${ }^{3}$ Clinical Trial Service Unit, University of Oxford, Oxford, United Kingdom

${ }^{4}$ Institute of Social and Preventive Medicine, University of Zurich, Zurich, Switzerland

${ }^{5}$ Department of Nutrition and Food Sciences, Nutritional Epidemiology, University of Bonn, Bonn, Germany

${ }^{6}$ CIBER Epidemiologia y Salud Publica (CIBERESP), Spain

${ }^{7}$ Public Health Division of Gipuzkoa, Instituto BIO-Donostia, Basque Government, Spain

${ }^{8}$ Department of Epidemiology and Biostatistics, School of Public Health, Imperial College London, London, United Kingdom

${ }^{9}$ International Agency for Research on Cancer, Lyon, France

${ }^{10}$ Department of Cancer Epidemiology, German Cancer Research Center, Heidelberg, Germany

${ }^{11}$ Department of Medical Biosciences, Pathology, Umeå University, Umeå, Sweden

${ }^{12}$ Department of Epidemiology, German Institute of Human Nutrition Potsdam-Rehbrücke, Nuthetal, Germany

${ }^{13}$ Molecular Epidemiology Group, Max Delbrueck Center for Molecular Medicine (MDC), Berlin-Buch, Germany

${ }^{14}$ Department of Public Health, Section for Epidemiology, Aarhus University, Aarhus, Denmark

${ }^{15}$ Department of Cardiology, Aalborg University Hospital, Aalborg, Denmark

${ }^{16}$ Public Health Directorate, Asturias, Spain

${ }^{17}$ Unit of Nutrition, Environment and Cancer, Cancer Epidemiology Research Programme, Catalan Institute of Oncology (ICO), Barcelona, Spain

${ }^{18}$ Escuela Andaluza de Salud Pública, Granada, Spain

${ }^{19}$ Instituto de Investigación Biosanitaria de Granada, Granada, Spain

${ }^{20}$ Department of Epidemiology, Murcia Regional Health Council, Murcia, Spain

${ }^{21}$ Navarre Public Health Institute, Pamplona, Spain

${ }^{22}$ Clinical Gerontology Unit, University of Cambridge School of Clinical Medicine, Cambridge, United Kingdom

Key words: type 2 diabetes, prostate cancer, cohort study, androgens, insulin-like growth factor proteins

Abbreviations: AMPK: adenosine monophosphate-activated protein kinase; BMI: body mass index; CVD: cardiovascular disease; CI: confidence intervals; EPIC: European Prospective Investigation into Cancer and Nutrition; HbA1c: glycated hemoglobin; HR: hazard ratios; IGF-I: insulin-like growth factor-one; IGFBP-III: insulin-like growth factor binding protein-three; IARC: International Agency for Research on Cancer; PCPT: Prostate Cancer Prevention Trial; PLCO: Prostate Lung Colorectal and Ovarian Cancer Screening Trial; PSA: prostate-specific antigen; ProtecT: Prostate testing for cancer and Treatment; SHBG: sex hormone binding globulin; TNM: tumornode-metastasis; UK: United Kingdom

Additional Supporting Information may be found in the online version of this article.

Grant sponsor: Seventh Framework Programme of the European Union; Grant number: PIEF-GA-2010-276017; Grant sponsor: European Commission (DG-SANCO), International Agency for Research on Cancer, Danish Cancer Society (Denmark), Ligue contre le Cancer, Société 3M, Mutuelle Générale de l'Education Nationale, Institut National de la Santé et de la Recherche Medicale (France); Deutsche Krebshilfe, Deutsches Krebsforschungszentrum and Federal Ministry of Education and Research (Germany), Hellenic Health Foundation (Greece), Italian Association for Research on Cancer (AIRC) and National Research Council (Italy), Dutch Ministry of Public Health, Welfare and Sports (VWS), Netherlands Cancer Registry (NKR), LK Research Funds, Dutch Prevention Funds, Dutch ZON (Zorg Onderzoek Nederland), World Cancer Research Fund (WCRF); Statistics Netherlands (The Netherlands), Norwegian Cancer Society (Norway), Health Research Fund (FIS), Regional Governments of Andalucía, Asturias, Basque Country, Murcia and Navarra, ISCIII RETIC (Spain); Grant number: RD06/0020; Grant sponsor: Swedish Cancer Society, Swedish Scientific Council and Regional Government of Skåne and Västerbotten, Fundacion Federico SA (Sweden), Cancer Research United Kingdom, Medical Research Council (UK).

DOI: $10.1002 / \mathrm{ijc} .28989$

History: Received 28 Nov 2013; Accepted 17 Mar 2014; Online 26 May 2014

Correspondence to: Konstantinos K. Tsilidis, Cancer Epidemiology Unit, University of Oxford, Richard Doll Building, Roosevelt Drive, Oxford, OX3 7LF, United Kingdom, Tel.: +44-(0)-1865-289647, Fax: +44-(0)-1865-289610, E-mail: kostas.tsilidis@ceu.ox.ac.uk 


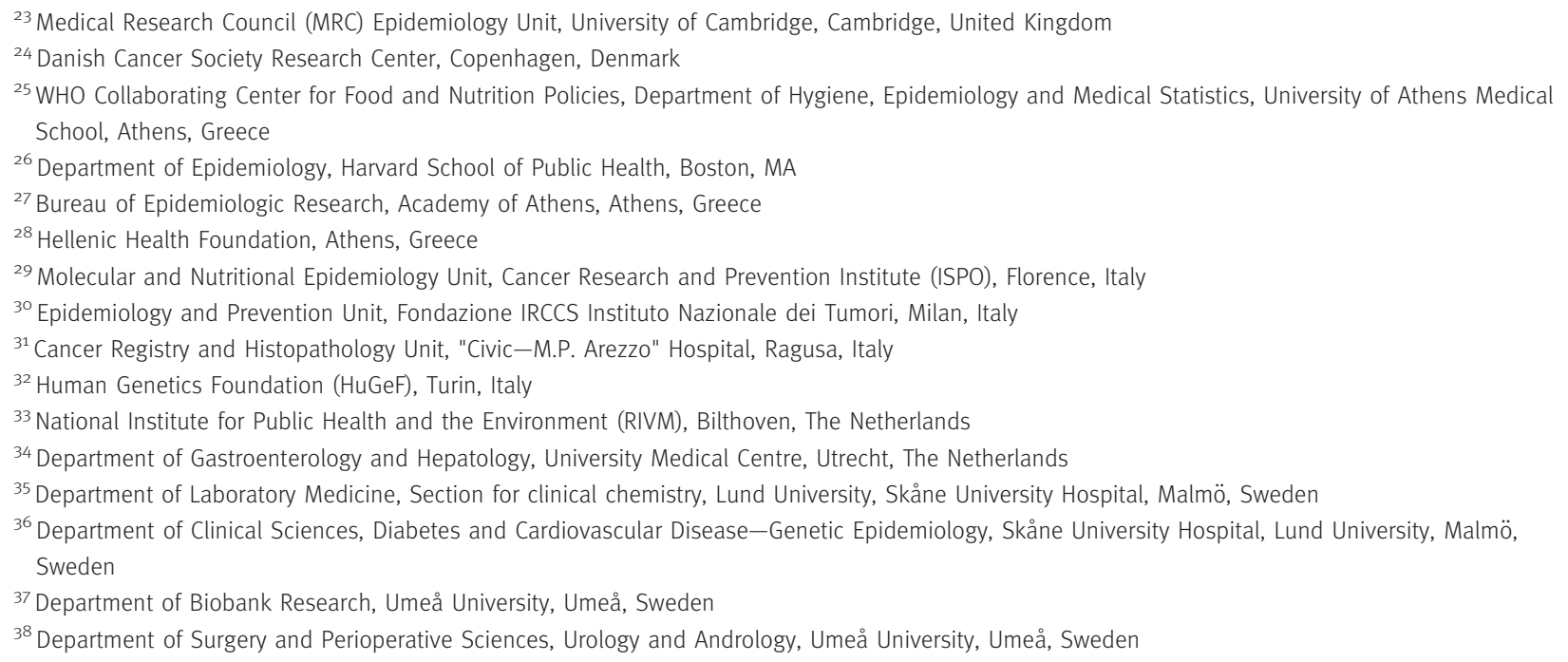

The current epidemiologic evidence suggests that men with type 2 diabetes mellitus may be at lower risk of developing prostate cancer, but little is known about its association with stage and grade of the disease. The association between selfreported diabetes mellitus at recruitment and risk of prostate cancer was examined in the European Prospective Investigation into Cancer and Nutrition (EPIC). Among 139,131 eligible men, 4,531 were diagnosed with prostate cancer over an average follow-up of 12 years. Multivariable hazard ratios (HR) and $95 \%$ confidence intervals (CI) were estimated using Cox proportional hazards models stratified by EPIC-participating center and age at recruitment, and adjusted for education, smoking status, body mass index, waist circumference, and physical activity. In a subset of men without prostate cancer, the crosssectional association between circulating concentrations of androgens and insulin-like growth factor proteins with diabetes status was also investigated using linear regression models. Compared to men with no diabetes, men with diabetes had a $26 \%$ lower risk of prostate cancer ( $\mathrm{HR}, 0.74 ; 95 \% \mathrm{Cl}, 0.63-0.86$ ). There was no evidence that the association differed by stage ( $p$-heterogeneity, 0.19$)$ or grade ( $p$-heterogeneity, 0.48$)$ of the disease, although the numbers were small in some disease subgroups. In a subset of 626 men with hormone measurements, circulating concentrations of androstenedione, total testosterone and insulin-like growth factor binding protein-three were lower in men with diabetes compared to men without diabetes. This large European study has confirmed an inverse association between self-reported diabetes mellitus and subsequent risk of prostate cancer.

\section{What's new?}

Emerging evidence suggests that men with type 2 diabetes are at lower risk to develop prostate cancer. Using data obtained within the European Prospective Investigation into Cancer and Nutrition (EPIC), the authors show that the prostate cancer risk was, indeed, reduced by $26 \%$ in men with type 2 diabetes but no association with cancer stage or grade was observed. In a subset of men for whom data on circulating hormones were available, levels of androstenedione, total testosterone and insulin-like growth factor binding protein-three were lower in those with diabetes as compared to those without diabetes, giving clues to how having diabetes could affect prostate cancer development.

Prostate cancer is the most frequently diagnosed cancer in men and one of the leading causes of cancer death in Europe and the United States. ${ }^{1}$ The only well-established risk factors for prostate cancer are age, ethnicity, family history of the disease and genetic factors.

Diabetes mellitus type 2 has been associated with a higher risk for several cancer types (e.g., liver, pancreas, endometrial, colorectal, bladder, non-Hodgkin lymphoma, breast). ${ }^{2}$ However, two recent meta-analyses reported a lower risk of total prostate cancer among men with diabetes, ${ }^{3,4}$ suggesting there is some unique aspect of prostate cancer pathophysiology or detection related to diabetes mellitus. For example, diabetes may lower circulating testosterone concentrations, known to be important in prostate growth and prostate cancer 
development. ${ }^{5}$ Alternatively, diabetes may be associated with lower prostate-specific antigen (PSA) concentrations, increased prostate size or a lower prevalence of PSA testing, leading to a reduction in the detection of localized biopsy-detected prostate cancer, although relatively few prospective studies have examined the association by stage and grade of the disease. ${ }^{5}$

The aim of the current study is to examine the association of diabetes mellitus with prostate cancer risk in the European Prospective Investigation into Cancer and Nutrition (EPIC). A secondary aim is to compare the circulating concentrations of androgens (androstenedione, androstanediol glucuronide, total and calculated free testosterone), sex hormone binding globulin (SHBG), insulin-like growth factor-one (IGF-I) and IGF binding protein-three (IGFBP-III) in men with and without diabetes in a subset of the cohort to explore the mechanisms through which diabetes might influence risk.

\section{Material and Methods \\ Study population}

EPIC is a prospective cohort with 23 centers in 10 European countries (Denmark, France, Germany, Greece, Italy, The Netherlands, Norway, Spain, Sweden, and the United Kingdom) that recruited more than half a million participants in the 1990s to investigate potential dietary, lifestyle, and serologic cancer risk factors. Participants were aged 35 to 70 years at enrolment between 1992 and 2000, and were voluntarily recruited predominantly from the general population residing in a given geographic area. Standardized lifestyle questionnaires, anthropometric data, and country-specific food questionnaires were collected via mail and a visit to an examination center from 521,457 participants, of whom 30\% were men. About 386,000 individuals also provided a $30 \mathrm{~mL}$ blood sample at recruitment drawn by experienced phlebotomists according to a standardized protocol, which was aliquoted at each EPIC center into $0.5 \mathrm{~mL}$ straws half of which was stored locally and the other half was transported to the International Agency for Research on Cancer (IARC) except for Sweden and Denmark where all samples were stored locally. In France and Norway only women were enrolled. All participants provided written informed consent. Approval for the study was obtained from the ethical review boards of the participating institutions and the (IARC). The cohort population and data collection procedures have been described in more detail elsewhere. ${ }^{6}$

The study population comprised 148,015 male participants after excluding men with prevalent cancer at recruitment. Men who did not return the baseline lifestyle and dietary questionnaire $(n=2,914)$, those who were in the top or bottom $1 \%$ of the ratio of energy intake to estimated energy requirement ( $n=2,847)$ and those with missing information on diabetes status at baseline $(n=3,123)$ were also excluded, leaving 139,131 men available for analysis.

\section{Assessment of prostate cancer}

Incident prostate cancer cases were identified through linkage to population cancer registries in Denmark, Italy, The Neth- erlands, Spain, Sweden, and the United Kingdom, or with a combination of methods including linkage to health insurance records, regional health departments, municipality registries, hospital- or physician-based cancer and pathology registries, and active follow-up through mail or phone calls to study participants or their next of kin in Germany and Greece. Follow-up began at the date of recruitment and ended at either the date of diagnosis of prostate cancer, death or last complete follow-up (from 31 December 2007 to 14 June 2010 according to center). A total of 4,531 men developed prostate cancer (International Classification of Diseases 10th revision codes: C61) in this time period.

Data on the stage and grade of prostate cancer at diagnosis were collected from each center, where possible. A total of 2,582 cases $(57 \%)$ had stage information, of which 1,688 were classified as localized (tumor-node-metastasis [TNM] staging score of $\mathrm{T}_{0}-\mathrm{T}_{2}$ and $\mathrm{N}_{0} / \mathrm{N}_{\mathrm{x}}$ and $\mathrm{M}_{0}$, or stage coded in the recruitment center as localized) and 894 were classified as advanced prostate cancer $\left(\mathrm{T}_{3}-\mathrm{T}_{4}\right.$ and/or $\mathrm{N}_{1}-\mathrm{N}_{3}$ and/or $\mathrm{M}_{1}$, or stage coded in the recruitment center as metastatic). A total of 2,186 cases ( $48 \%$ ) had grade information, of which 1,811 were classified as low-grade (Gleason score of $<8$, or grade coded as well differentiated or moderately differentiated) and 375 were classified as high-grade prostate cancer (Gleason score of $\geq 8$, or grade coded as poorly differentiated or undifferentiated). When a Gleason score of 7 (instead of 8) was used to define prostate cancer grade in a sensitivity analysis, the associations between diabetes and cancer grade were very similar (data not shown).

\section{Assessment of diabetes and other variables}

Information on socio-demographics, lifestyle characteristics, anthropometric variables and dietary intakes were collected via questionnaires at the time of recruitment. Information on medical history was also collected, including whether participants had ever been diagnosed with diabetes and if so, at what age. No distinction was made between type 1 or type 2 diabetes, although the vast majority (93\%) were diagnosed after the age of 30 and are therefore likely to be type 2 diabetes. Information on the use of insulin treatment was selfreported from the baseline questionnaire. Some EPIC centers subsequently validated the self-reported diagnosis of diabetes at baseline by cross-referencing with additional information sources (sensitivity of 79\%) including: verification by a medical practitioner, use of diabetes medication, repeated selfreport of diabetes diagnosis in follow-up questionnaires, linkage to diabetes registries, or a glycated hemoglobin (HbA1c) concentration of above $6 \%(42 \mathrm{mmol} / \mathrm{mol}){ }^{7}$

Weight, height, waist and hip circumference were measured at recruitment, except for part of the Oxford cohort, where height and weight were self-reported. Body mass index (BMI) was calculated as weight in kilograms divided by height in meters squared. Food and nutrient intakes were estimated from the baseline food questionnaire, details of which are published elsewhere. ${ }^{6}$ 
All laboratory assays were performed at IARC from blood samples collected at recruitment and stored at liquid nitrogen $\left(-196^{\circ} \mathrm{C}\right)$ since then, except for the most recent IGF assays that were measured at the Cancer Epidemiology Unit, University of Oxford, by experienced personnel who were blinded to the case-control or diabetic status of the blood samples. Androstenedione, androstanediol glucuronide, testosterone and SHBG concentrations were measured by radio-immunoassays. Free testosterone concentrations were calculated using the law of mass action from the measured values of testosterone and SHBG, assuming a constant serum albumin concentration of $43 \mathrm{~g} / \mathrm{L} .{ }^{8}$ Serum IGF-I and IGFBP-III concentrations were measured by ELISAs. More details on blood collection and processing, assay methods and quality control statistics are described elsewhere. $^{9,10}$

\section{Statistical analysis}

Cox proportional hazards models were used to estimate hazard ratios (HR) and 95\% confidence intervals (95\% CI) for prostate cancer using age as the underlying time scale. Age at entry was defined as the participants' age at recruitment, and exit time was age at diagnosis of prostate cancer, death, loss to follow-up or censoring at the end of the follow-up period, whichever came first. The proportionality of hazards was verified based on the slope of the Schoenfeld residuals over time. Linear trends for time since diabetes diagnosis were tested by entering an ordinal variable into the models, the coefficients of which were evaluated by the Wald test. All models were stratified by EPIC recruitment center and age at enrolment $(<50$, $50-54,55-59,60-64,65-69, \geq 70$ years). Models were adjusted for known or suspected risk factors for prostate cancer, including education (less than university, university graduate), smoking status (never, former, current), BMI (in quintiles: $<23.6, \geq 23.6<25.5, \geq 25.5<27.2, \geq 27.2<29.4, \geq 29.4$ $\mathrm{kg} / \mathrm{m}^{2}$ ), waist circumference (in quintiles: $<86, \geq 86<92$, $\geq 92<97, \geq 97<103, \geq 103 \mathrm{~cm}$ ), and physical activity (inactive, moderately inactive, moderately active, active). Missing values were assigned to separate categories for education (2.6\%), smoking status (1.4\%), BMI $(0.6 \%)$, waist circumference $(9.7 \%)$, and physical activity (2.2\%), and missing indicators were used in the statistical models. Analyses that excluded men with missing values for any of these covariates, and analyses that included further adjustments for employment, hip circumference, waist to hip ratio, dietary intake of energy, alcohol, fruits and vegetables, fish, protein from dairy sources, calcium, red meat intake, and self-reported history of cardiovascular disease (CVD) gave very similar results and are not presented here.

Stratified analyses were conducted according to age at recruitment $(<60$ vs. $\geq 60$ years $)$, BMI $(<25,25-29$, $\geq 30 \mathrm{~kg} / \mathrm{m}^{2}$ ), and physical activity (inactive and moderately inactive $v s$. moderately active and active), because these variables are known to influence diabetes and/or prostate cancer risk. Tests for interaction were carried out by using the rele- vant exposure variables, indicator variables for the potentially modifying factors, and product terms of the two variables. The statistical significance of the interaction terms was evaluated by the Wald test. Country-specific analyses were also performed. All $p$ values $(p)$ were two-sided and all analyses were performed using STATA version 12 (College Station, TX).

Sensitivity analyses were performed that (i) excluded men with possible type 1 diabetes due to their potentially different metabolic profile and thus different aetiology ( $n=339$ ); (ii) restricted analyses to men with a validated diagnosis of diabetes $(n=3,202)$; (iii) excluded men with a self-reported history of CVD $(n=30,918)$ and those with missing CVD information to approximate treatment with a statin $(n=$ 5,045); (iv) excluded men diagnosed with prostate cancer in the first three years after the diagnosis of diabetes, due to the potential for heightened medical surveillance, but no such cases existed in our cohort.

To shed light on the association linking diabetes with prostate cancer, we evaluated the cross-sectional association of naturally logarithm-transformed androgen concentrations (androstenedione, androstanediol glucuronide, total and calculated free testosterone), SHBG, IGF-I and IGFBP-III with diabetes status using a linear regression model controlling for laboratory batch, EPIC center, age at recruitment, and BMI among 626 men without prostate cancer (controls) for whom measurements were available from published nested casecontrol studies in EPIC that evaluated the association between these hormones and risk of prostate cancer. ${ }^{9,10}$ The IGF-I analysis was conducted in 1,527 men.

\section{Results}

Overall, 139,131 men were followed-up for an average of 12 years (range, 0-18 years), of whom 4,531 men developed prostate cancer (Table 1). The overall mean age at recruitment was 52 years, and ranged from 43 years in the Netherlands to 56 years in Denmark, while the overall mean age at prostate cancer diagnosis was 67 years. The proportion of men with self-reported diabetes at recruitment was $3.7 \%$ (5,100 men), and ranged from $1.2 \%$ in the Netherlands to $7.1 \%$ in Greece. A total of $1,546(30 \%)$ diabetic men had a duration of diabetes at recruitment of more than 10 years, and use of insulin treatment was reported by 813 (16\%) diabetic men.

The age-adjusted characteristics of the men with and without diabetes are shown in Table 2. Compared to men without a history of diabetes, men with diabetes were on average older (mean age: 51.4 vs. 57.5 years), had lower educational level (\% university graduate: 27.2 vs. 21.9), were less likely to be in paid employment (81.2 vs. $71.2 \%)$, less physically active (24.6 vs. $18.8 \%)$, had a greater self-reported history of CVD (21.2 vs. $40.4 \%$ ), and had a higher BMI (mean: 26.6 vs. $28.1 \mathrm{~kg} / \mathrm{m}^{2}$ ) but reported a lower caloric intake (mean: 2414 vs. $2253 \mathrm{kcal}$ ). 


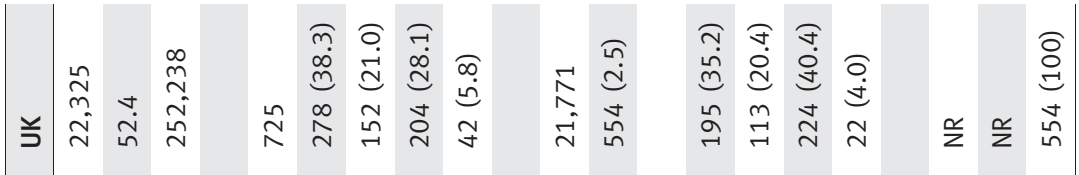

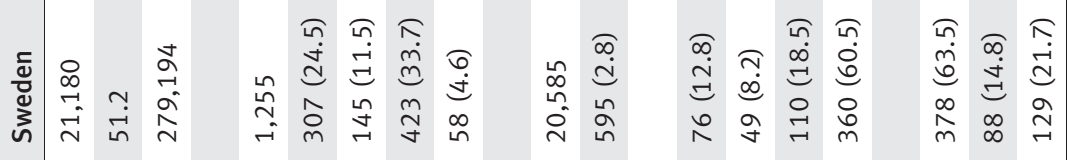

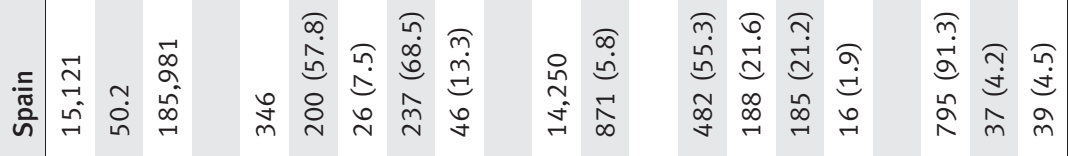

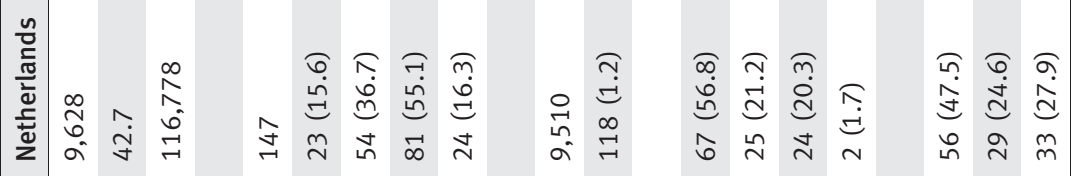

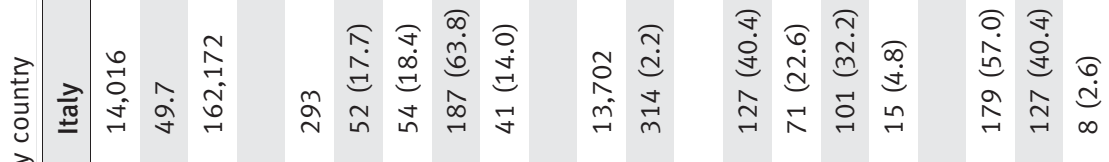

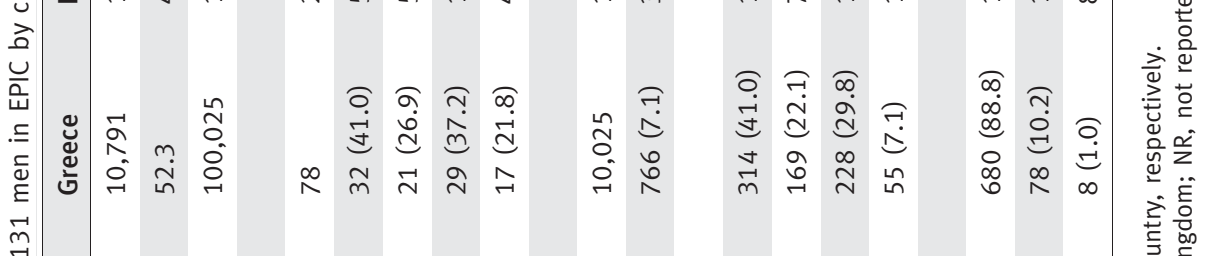

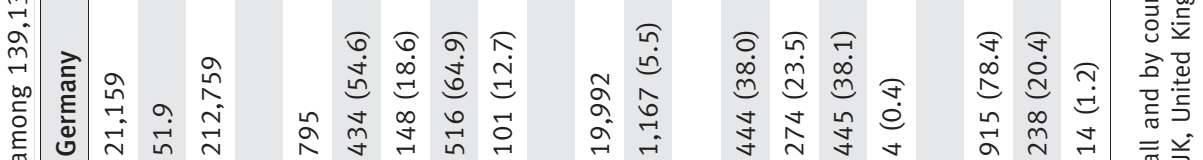

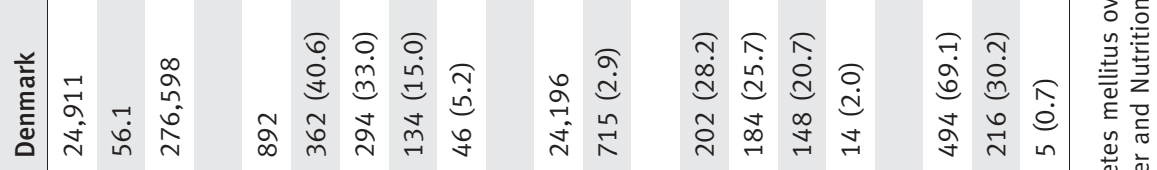

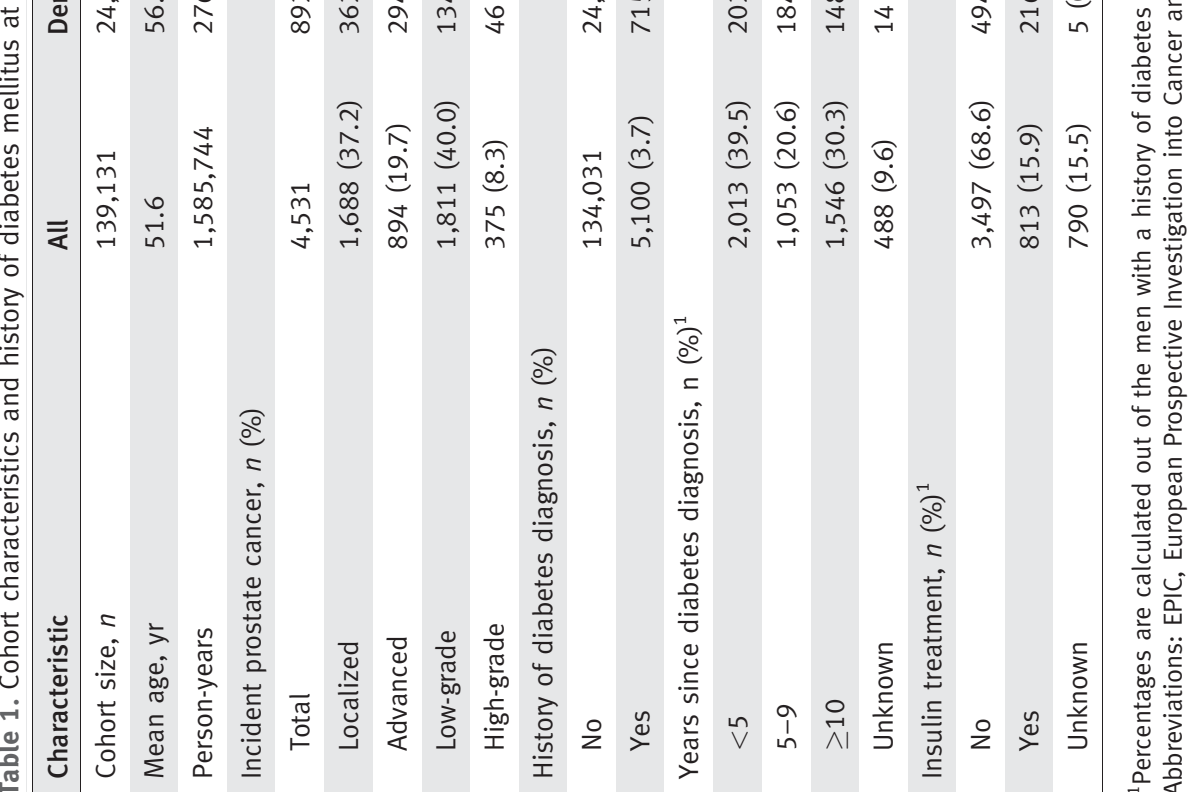


Table 2. Selected characteristics at recruitment in relation to history of diabetes mellitus among 139,131 men in EPIC ${ }^{1}$

\begin{tabular}{lll}
\hline & \multicolumn{2}{c}{ History of diabetes mellitus } \\
\cline { 2 - 3 } Characteristic & No $(n=134,031)$ & Yes $(n=5,100)$ \\
\hline Mean age (SD), yr & $51.4(10.1)$ & $21.9(20.7-23.1)$ \\
\hline University graduate $(95 \% \mathrm{Cl}), \%$ & $27.2(26.9-27.4)$ & $71.2(69.8-72.6)$ \\
\hline Paid employment $(95 \% \mathrm{Cl}), \%$ & $81.2(80.9-81.5)$ & $30.2(28.9-31.5)$ \\
\hline Current smoker $(95 \% \mathrm{Cl}), \%$ & $29.7(29.4-29.9)$ & $18.8(17.6-19.9)$ \\
\hline Physically active $(95 \% \mathrm{Cl}), \%$ & $24.6(24.4-24.9)$ & $40.4(39.0-41.8)$ \\
\hline Self-reported cardiovascular disease $(95 \% \mathrm{Cl}), \%$ & $21.2(21.0-21.5)$ & $28.1(28.0-28.2)$ \\
\hline Mean body mass index $(95 \% \mathrm{Cl}), \mathrm{kg} / \mathrm{m}^{2}$ & $26.6(26.5-26.6)$ & $99.0(98.7-99.2)$ \\
\hline Mean waist circumference $(95 \% \mathrm{Cl}), \mathrm{cm}$ & $94.8(94.7-94.8)$ & $103(102-103)$ \\
\hline Mean hip circumference $(95 \% \mathrm{Cl}), \mathrm{cm}$ & $101(101-102)$ & $0.96(0.96-0.97)$ \\
\hline Mean waist to hip ratio $(95 \% \mathrm{Cl})$ & $0.94(0.93-0.94)$ & \\
\hline Daily mean $(95 \% \mathrm{Cl})$ dietary intake & & $2,253(2,235-2,271)$ \\
\hline Energy, kcal & $2,414(2,411-2,418)$ & $20.6(20.0-21.2)$ \\
\hline Alcohol, g & $20.8(20.7-20.9)$ & $474(467-481)$ \\
\hline Fruits and vegetables, g & $399(397-400)$ & $60.1(59.1-61.2)$ \\
\hline Red meat, g & $58.5(58.3-58.7)$ & $32.8(32.1-33.6)$ \\
\hline Fish, g & $27.3(27.2-27.5)$ & $1,106(1,096-1,116)$ \\
\hline Calcium, mg & $1,039(1,037-1,041)$ & $20.9(20.6-21.2)$ \\
\hline Protein from dairy sources, $\mathrm{g}$ & $19.2(19.1-19.3)$ & \\
\hline
\end{tabular}

${ }^{1}$ Age at recruitment-adjusted means and proportions are presented using linear and logistic regression models, respectively. The means of dietary intake are also adjusted for daily energy intake and EPIC center.

Abbreviations: EPIC, European Prospective Investigation into Cancer and Nutrition; SD, standard deviation; Cl, confidence interval.

\section{Diabetes and total prostate cancer}

Of the 4,531 prostate cancer cases, 157 (3.5\%) men had a self-reported history of diabetes at recruitment. A selfreported history of diabetes was associated with a $27 \%$ reduced risk of total prostate cancer (Table 3; HR, 0.73; 95\% CI, 0.62-0.85). Additional adjustment for education, smoking, BMI, waist circumference and physical activity made little difference to this risk estimate (HR, 0.74; 95\% CI, 0.63-0.86). Compared to men who were diagnosed with diabetes less than 5 years before recruitment, men with longer-standing diabetes did not have a lower risk for total prostate cancer $(\geq 10$ vs. $<5$ years since diabetes diagnosis; HR, 0.99; 95\% CI, 0.66-1.48; based on 134 cases). However, when compared to men without diabetes, the association was lower with increasing duration of diabetes $(>12$ years since diabetes diagnosis vs. no history of diabetes; HR, 0.64; 95\% CI, 0.45-0.92; $p$ trend, 0.001). Compared to men with diabetes not on insulin treatment, insulin was not associated with risk of total prostate cancer (HR, 0.86; 95\% CI, 0.511.48) and further adjustment for duration of diabetes did not alter the results (data not shown). The association of diabetes status and prostate cancer risk did not change according to subgroups of age at recruitment, BMI, physical activity (Supporting Information Table S1) or by country ( $p$-heterogeneity, 0.81).

\section{Diabetes and prostate cancer by stage and grade}

There was no statistical evidence (Table 3) that the association between self-reported history of diabetes and prostate cancer risk differed by stage or grade of the disease ( $p$-heterogeneity of 0.19 and 0.48 , respectively), although diabetes was associated with a $30 \%$ reduced risk of localized prostate cancer (HR, 0.70; 95\% CI, 0.54-0.90; based on 63 cases with diabetes), and was not associated with advanced disease (HR, 0.93; 95\% CI, 0.66-1.30; based on 36 cases with diabetes). For grade, diabetes was associated with a $19 \%$ reduced risk of low-grade prostate cancer (HR, 0.81; 95\% CI, 0.64-1.01; based on 80 cases with diabetes), and a $34 \%$ lower risk of high-grade disease (HR, 0.66; 95\% CI, 0.39-1.12; based on 15 cases with diabetes).

\section{Sensitivity analyses}

Sensitivity analyses that excluded men with possible type 1 diabetes (i.e., 339 men with an age at diabetes diagnosis below 30 years) did not change the overall risk estimate (HR, 0.74; 95\% CI, 0.62-0.87). Similarly, when analyses were restricted to the 3,202 men with a verified diabetes diagnosis, the corresponding risk estimate was 0.79 (95\% CI, 0.66$0.94)$. When the analysis was restricted to 103,168 men without a self-reported history of cardiovascular disease, the inverse association of diabetes with prostate cancer risk became slightly stronger (HR, 0.63; 95\% CI, 0.50-0.81). 
Table 3. Hazard ratio (HR) and 95\% confidence interval (CI) for diabetes mellitus and prostate cancer among 139,131 men in EPIC

\begin{tabular}{|c|c|c|c|c|c|}
\hline Variable & Cases & Person-years & $\begin{array}{c}\text { Age and center-stratified, } \\
\text { HR }(95 \% \mathrm{Cl})\end{array}$ & $\begin{array}{c}\text { Multivariable adjusted, } \\
\text { HR }(95 \% \mathrm{Cl})^{1}\end{array}$ & $p$-heterogeneity \\
\hline \multicolumn{6}{|c|}{ Total prostate cancer } \\
\hline \multicolumn{6}{|c|}{ History of diabetes } \\
\hline No & 4,374 & $1,498,428$ & 1.00 (reference) & 1.00 (reference) & \\
\hline Yes & 157 & 51,794 & $0.73(0.62-0.85)$ & $0.74(0.63-0.86)$ & \\
\hline \multicolumn{6}{|c|}{ Years since diabetes } \\
\hline$<5$ & 55 & 20,788 & 1.00 (reference) & 1.00 (reference) & \\
\hline $5-9$ & 30 & 10,493 & $1.02(0.65-1.61)$ & $1.01(0.64-1.60)$ & \\
\hline$\geq 10$ & 49 & 15,135 & $0.99(0.66-1.47)$ & $0.99(0.66-1.48)$ & \\
\hline$p$-trend & & & 0.96 & 0.97 & \\
\hline \multicolumn{6}{|c|}{ Insulin treatment } \\
\hline No & 113 & 35,530 & 1.00 (reference) & 1.00 (reference) & \\
\hline Yes & 20 & 7,875 & $0.84(0.50-1.43)$ & $0.86(0.51-1.48)$ & \\
\hline \multicolumn{6}{|c|}{ Localized prostate cancer $^{2}$} \\
\hline \multicolumn{6}{|c|}{ History of diabetes } \\
\hline No & 1,625 & $1,498,428$ & 1.00 (reference) & 1.00 (reference) & \\
\hline Yes & 63 & 51,794 & $0.69(0.54-0.89)$ & $0.70(0.54-0.90)$ & \\
\hline \multicolumn{6}{|c|}{ Advanced prostate cancer ${ }^{3}$} \\
\hline \multicolumn{6}{|c|}{ History of diabetes } \\
\hline No & 858 & $1,498,428$ & 1.00 (reference) & 1.00 (reference) & \\
\hline Yes & 36 & 51,794 & $0.92(0.66-1.29)$ & $0.93(0.66-1.30)$ & 0.19 \\
\hline \multicolumn{6}{|c|}{ Low-grade prostate cancer ${ }^{4}$} \\
\hline \multicolumn{6}{|c|}{ History of diabetes } \\
\hline No & 1,731 & $1,498,428$ & 1.00 (reference) & 1.00 (reference) & \\
\hline Yes & 80 & 51,794 & $0.79(0.63-0.99)$ & $0.81(0.64-1.01)$ & \\
\hline \multicolumn{6}{|c|}{ High-grade prostate cancer ${ }^{5}$} \\
\hline \multicolumn{6}{|c|}{ History of diabetes } \\
\hline No & 360 & $1,498,428$ & 1.00 (reference) & 1.00 (reference) & \\
\hline Yes & 15 & 51,794 & $0.69(0.41-1.16)$ & $0.66(0.39-1.12)$ & 0.48 \\
\hline
\end{tabular}

${ }^{1}$ From a Cox proportional hazards model stratified by EPIC-participating center and age at recruitment, and adjusted for education, smoking status, body mass index, waist circumference, and physical activity.

${ }^{2}$ Tumor-node-metastasis staging score of $\mathrm{T}_{0}-\mathrm{T}_{2}$ and $\mathrm{N}_{0} / \mathrm{N}_{\mathrm{x}}$ and $\mathrm{M}_{0}$, or stage coded in the recruitment center as localized.

${ }^{3} \mathrm{~T}_{3}-\mathrm{T}_{4}$ and/or $\mathrm{N}_{1}-\mathrm{N}_{3}$ and/or $\mathrm{M}_{1}$, or stage coded in the recruitment center as metastatic.

${ }^{4}$ Gleason score of $<8$, or grade coded as well differentiated or moderately differentiated.

${ }^{5}$ Gleason score of $\geq 8$, or grade coded as poorly differentiated or undifferentiated.

\section{Sex steroid hormones, IGFs and diabetes}

To investigate potential mechanisms that could explain the inverse association between diabetes and prostate cancer in our study population, we evaluated the association between circulating concentrations of several sex hormones and growth factors previously implicated in prostate cancer etiology according to diabetes status (Table 4). Compared to men without diabetes, men with diabetes had lower concentrations of androstenedione (geometric mean: 4.16 vs. $4.72 \mathrm{nmol} / \mathrm{L} ; p$, 0.03 ), total testosterone (14.1 vs. $15.9 \mathrm{nmol} / \mathrm{L} ; p, 0.09)$, and IGFBP-III (117 vs. $130 \mathrm{nmol} / \mathrm{L} ; p$, 0.006), but the sample size was small in some of these cross-sectional analyses.

\section{Discussion}

In this cohort study involving 139,131 men and 4,531 incident prostate cancers, there was a $26 \%$ reduction in risk of prostate cancer associated with a self-reported history of diabetes. There was no evidence that the association between diabetes and prostate cancer varied significantly by stage and grade of the disease, although there were small numbers of men with diabetes and resulting low statistical power in some disease subgroups.

Previous meta-analyses have also reported a lower risk of prostate cancer among men with diabetes, the most recent of which included 45 studies and observed a statistically 
Table 4. Geometric means and $95 \%$ confidence intervals of androgen and growth factor concentrations by diabetic status in a subset of EPIC controls $^{1}$

\begin{tabular}{|c|c|c|c|}
\hline \multirow[b]{3}{*}{ Androgens and growth factors } & \multicolumn{3}{|c|}{ History of diabetes ${ }^{2}$} \\
\hline & No & Yes & \\
\hline & Mean $(95 \% \mathrm{Cl})$ & Mean $(95 \% \mathrm{Cl})$ & $\mathrm{p}$ \\
\hline Androstenedione (nmol/L) & $4.72(4.60-4.84)$ & $4.16(3.73-4.64)$ & 0.03 \\
\hline Androstanediol glucuronide (nmol/L) & $12.9(12.3-13.5)$ & $11.7(9.69-14.2)$ & 0.35 \\
\hline Total testosterone (nmol/L) & $15.9(15.4-16.4)$ & $14.1(12.3-16.2)$ & 0.09 \\
\hline Free testosterone (pmol/L) & $273(265-282)$ & $244(213-280)$ & 0.12 \\
\hline Sex hormone binding globulin (nmol/L) & $43.3(41.9-44.8)$ & $39.6(34.4-45.6)$ & 0.22 \\
\hline Insulin-like growth factor-I (nmol/L) & $19.8(19.5-20.1)$ & $19.4(18.0-20.9)$ & 0.58 \\
\hline Insulin-like growth factor binding protein-III (nmol/L) & $130(128-132)$ & $117(109-126)$ & 0.006 \\
\hline
\end{tabular}

${ }^{1}$ From a linear regression analysis between natural log-transformed androgen and growth factor concentrations and diabetic status at baseline adjusted for laboratory batch, age at blood draw, body mass index, EPIC center.

${ }^{2}$ All analyses were conducted among 37 diabetic and 589 non-diabetic controls, except for insulin-like growth factor-I analysis where 78 diabetic and 1,449 non-diabetic controls were used.

Abbreviations: $\mathrm{Cl}$, confidence interval.

significant $14 \%$ risk reduction. ${ }^{4}$ An inverse association was also observed in the Prostate Cancer Prevention Trial (PCPT), where all men underwent biopsy at the end of the study, thereby reducing the possibility of detection bias. ${ }^{11}$ Very few studies have not reported an inverse association, and they had either a short follow-up, few incident cancer events or did not adjust for obesity. ${ }^{12-14}$ However, a recent report from the REDUCE trial, where all men had biopsies both during and at the end of the study, showed a null association between diabetes and subsequent prostate cancer incidence, ${ }^{15}$ although this study had only a 4 -year follow-up and likely included men with better-controlled diabetes. Further evidence supporting the notion that the inverse association between diabetes and prostate cancer may be causal comes from studies where the association appears to strengthen with increasing duration of diabetes compared to men without diabetes, which agree with our findings. ${ }^{16-20}$ However, when we performed a more appropriate linear trend analysis only among men with diabetes, longer duration of the disease was not associated with the risk of prostate cancer but this analysis was based on 134 cases and has not been performed in other studies. Only this analysis may directly identify the association of diabetes duration on cancer risk over and beyond the effect of diabetes diagnosis per se and also men with diabetes may differ from men without the disease in several characteristics that may not be appropriately captured in any list of confounders. Recent genetic studies have highlighted a potential genetic link between diabetes mellitus and prostate cancer. The A allele of a single nucleotide polymorphism (rs4430796) in the hepatocyte nuclear factor-1 $\beta$ gene was associated with an increased risk of prostate cancer, whereas the same allele of the same variant was inversely associated with type 2 diabetes in a genome-wide association study. $^{21}$ In addition, diabetes mellitus susceptibility genetic scores have been inversely associated with prostate cancer, ${ }^{22}$ but this finding is not entirely consistent. ${ }^{23}$
Several potential mechanisms have been proposed to explain the inverse association between diabetes mellitus and prostate cancer. First, men with severe type 2 diabetes have lower circulating testosterone concentrations, possibly resulting from the detrimental effect of hyperglycemia on testosterone production. ${ }^{24}$ While our finding of lower testosterone concentrations in men with diabetes supports this hypothesis, it is uncertain whether this would directly impact prostate cancer development, as the current epidemiologic evidence does not support an association between adult circulating testosterone levels in the normal range and prostate cancer risk. ${ }^{25}$ However, randomized clinical trials and epidemiological studies have shown that decreased intraprostatic androgenic stimulation with use of $5 \alpha$-reductase inhibitors can decrease the risk of total prostate cancer, ${ }^{26}$ although it is unknown whether diabetes affects intraprostatic androgen concentrations. Other potential proposed mechanisms for the inverse association between diabetes and prostate cancer include the relative hypoinsulinemia after long-standing diabetes, as insulin levels decrease over time with pancreatic beta cell depletion, and the resulting limited bioavailability of IGF-I through modulating IGF-binding proteins, ${ }^{27}$ which has been associated with a decreased risk of prostate cancer in epidemiological studies. ${ }^{28}$ However, IGF-I was not lower in diabetics compared to non-diabetics in our study but a recent prospective investigation reported an inverse association between free IGF-I and risk of diabetes. ${ }^{29}$

Data from the PCPT and the ProtecT (Prostate testing for cancer and Treatment) studies suggested that the inverse association with diabetes was stronger for low grade tumors compared to high grade disease, but the associations were similar by stage. ${ }^{11,30}$ In addition, the Prostate, Lung, Colorectal, and Ovarian Cancer Screening Trial (PLCO) observed that the inverse association with diabetes was present for localized and less aggressive tumors but not for advanced or more aggressive disease. ${ }^{31}$ However, several other studies found no such differences by tumour 
aggressiveness, ${ }^{16,17,20}$ which is consistent with our findings, together with a meta-analysis of nine studies. ${ }^{32}$ Given the relatively small number of studies with available stage and grade information and the small proportion of advanced tumors in our study and in populations with screen-detected cases, further prospective epidemiological studies are needed to confirm the association of diabetes with different stages and grades of prostate cancer and also explore the association with lethal disease.

The association between type 2 diabetes and risk of prostate cancer is complicated by the several types of drug therapy often necessary for diabetes treatment. Potential protective effects of diabetes medications could also contribute to the inverse association between diabetes and prostate cancer. The biguanide metformin is the most commonly used first-line therapy for type 2 diabetes persons. Metformin activates the liver enzyme adenosine monophosphate-activated protein kinase (AMPK), which has been shown to inhibit growth of cancer cells in several in vitro and in vivo studies. ${ }^{33}$ The upstream regulator of AMPK is the protein kinase LKB1, which is a well-recognized tumor suppressor. Treatment with metformin has been associated in some studies with a reduced risk of total cancer incidence or mortality, but results from clinical trials or observational studies have generally not found a reduced risk of prostate cancer. ${ }^{34,35}$ While EPIC has information on insulin treatment at recruitment, it does not have data on other treatments, which may limit our interpretation of the cross-sectional analyses of hormone concentrations by diabetes status, as diabetes medication may influence IGF and sex hormone bioavailability. ${ }^{36}$

In interpreting the observed inverse association between diabetes and prostate cancer, it is important to consider noncausal explanations that could have been generated by various potential biases. First, most but not all studies have shown that diabetes may indirectly suppress PSA values, ${ }^{37-39}$ which could mean that men with diabetes are less likely to be detected with localized disease compared to men without diabetes. However, overall there is no evidence that diabetes is more strongly associated with aggressive disease. Furthermore, studies that have either adjusted for PSA screening practices or that have biopsied all participants at the study end (thereby minimizing PSA-mediated detection bias) have still observed inverse associations. ${ }^{11,16}$ Second, diabetes may increase the risk for an enlarged prostate (benign prostatic hyperplasia), and thus lead to a decreased likelihood to detect cancer with biopsy. ${ }^{38}$ However, the evidence supporting this detection bias is not consistent, because prostate size has been linearly and positively associated with serum concentrations of PSA, ${ }^{37}$ high values of which would increase the like- lihood of receiving a biopsy. Moreover, participants with enlarged prostates were excluded from the PCPT that still observed a significant inverse association between diabetes and prostate cancer. ${ }^{11}$ Third, a study has showed that diabetics were less likely to be screened with PSA, ${ }^{40}$ but other studies indicated no significant differences. ${ }^{17,31}$ Fourth, diabetes classification was based on self-report in our study and misclassification of patients with diabetes who did not know that they had the disease to the referent group of participants without diabetes is likely. However, any misclassification would have been non-differential, as the assessment of diabetes was performed at baseline long before cancer diagnosis, and thus it would have attenuated our results towards the null rather than be responsible for the observed inverse association. Moreover, self-reported measures of diabetes status have been previously shown to be $99.7 \%$ specific and $66 \%$ sensitive when compared to medical records. ${ }^{41}$

Residual confounding for known or suspected risk factors for prostate cancer is unlikely to explain the inverse association, because adjustment for a wide range of measured covariates made no material difference to our observed results. However, long-standing type 2 diabetics have a high prevalence of dyslipidemia and may be at increased risk for cardiovascular disease, therefore a large number of them will also be treated with a lipid-lowering medication such as a statin. Although the overall evidence for an association between statin use and prostate cancer is not strong, a recent metaanalysis showed that statins may be associated with a small reduction in the risk of total prostate cancer, ${ }^{42}$ another study also showed an inverse association between use of metformin and prostate cancer only among the subgroup of diabetics concurrently taking statins. ${ }^{43}$ We did not have information on statin use in EPIC, but adjusting for self-reported history of cardiovascular disease did not materially alter our risk estimates. Furthermore, when participants with a history of cardiovascular disease were excluded, the inverse association for diabetes and prostate cancer was strengthened, suggesting that residual confounding from statin use is unlikely to explain our findings.

In conclusion, our study confirmed an inverse association between diabetes mellitus and risk of prostate cancer. More work is needed to improve understanding of the effects of diabetes, its duration and its subsequent treatment on sex hormone and IGF concentrations and risk of prostate cancer.

\section{Acknowledgements}

This work was supported by the seventh framework programme of the European Union (PIEF-GA-2010-276017 to K.K.T.).

\section{References}

1. American Cancer Society. Global cancer facts and figures 2007. American Cancer Society, Atlanta, GA 2007.

2. Vigneri P, Frasca F, Sciacca L, et al. Diabetes and cancer. Endocr Relat Cancer 2009;16:1103-23.
3. Kasper JS, Giovannucci E. A meta-analysis of diabetes mellitus and the risk of prostate cancer. Cancer Epidemiol Biomarkers Prev 2006;15:2056-62.

4. Bansal D, Bhansali A, Kapil G, et al. Type 2 diabetes and risk of prostate cancer: a meta-analysis of observational studies. Prostate Cancer Prostatic Dis 2013;16:151-8.

5. Pierce BL. Why are diabetics at reduced risk for prostate cancer? A review of the epidemiologic evidence. Urol Oncol 2012;30:735-43. 
6. Riboli E, Hunt KJ, Slimani N, et al. European Prospective Investigation into Cancer and Nutrition (EPIC): study populations and data collection. Public Health Nutr 2002;5:1113-24.

7. Sluik D, Boeing H, Montonen J, et al. Associations between general and abdominal adiposity and mortality in individuals with diabetes mellitus. Am J Epidemiol 2011;174:22-34.

8. Sodergard R, Backstrom T, Shanbhag V, et al. Calculation of free and bound fractions of testosterone and estradiol-17 beta to human plasma proteins at body temperature. J Steroid Biochem 1982;16:801-10.

9. Travis RC, Key TJ, Allen NE, et al. Serum androgens and prostate cancer among 643 cases and 643 controls in the European Prospective Investigation into Cancer and Nutrition. Int J Cancer 2007;121:1331-8.

10. Allen NE, Key TJ, Appleby PN, et al. Serum insulin-like growth factor (IGF)-I and IGFbinding protein-3 concentrations and prostate cancer risk: results from the European Prospective Investigation into Cancer and Nutrition. Cancer Epidemiol Biomarkers Prev 2007;16:11217.

11. Gong Z, Neuhouser ML, Goodman PJ, et al. Obesity, diabetes, and risk of prostate cancer: results from the prostate cancer prevention trial. Cancer Epidemiol Biomarkers Prev 2006;15:1977-83.

12. Li Q, Kuriyama S, Kakizaki M, et al. History of diabetes mellitus and the risk of prostate cancer: the Ohsaki Cohort Study. Cancer Causes Control 2010;21:1025-32.

13. Tseng $\mathrm{CH}$. Diabetes and risk of prostate cancer: a study using the National Health Insurance. Diabetes Care 2011;34:616-21.

14. Lee MY, Lin KD, Hsiao PJ, et al. The association of diabetes mellitus with liver, colon, lung, and prostate cancer is independent of hypertension, hyperlipidemia, and gout in Taiwanese patients. Metabolism 2012;61:242-9.

15. Wu C, Moreira DM, Gerber L, et al. Diabetes and prostate cancer risk in the REDUCE trial. Prostate Cancer Prostatic Dis 2011;14:326-31.

16. Kasper JS, Liu Y, Giovannucci E. Diabetes mellitus and risk of prostate cancer in the health professionals follow-up study. Int J Cancer 2009;124: 1398-403.

17. Calton BA, Chang SC, Wright ME, et al. History of diabetes mellitus and subsequent prostate cancer risk in the NIH-AARP Diet and Health Study. Cancer Causes Control 2007;18:493-503.

18. Zhu K, Lee IM, Sesso HD, et al. History of diabetes mellitus and risk of prostate cancer in physicians. Am J Epidemiol 2004;159:978-82.
19. Atchison EA, Gridley G, Carreon JD, et al. Risk of cancer in a large cohort of U.S. veterans with diabetes. Int J Cancer 2011;128:635-43.

20. Fall K, Garmo H, Gudbjornsdottir S, et al. Diabetes mellitus and prostate cancer risk; a nationwide case-control study within PCBaSe Sweden. Cancer Epidemiol Biomarkers Prev 2013;22:1102-9.

21. Gudmundsson J, Sulem P, Steinthorsdottir V, et al. Two variants on chromosome 17 confer prostate cancer risk, and the one in TCF 2 protects against type 2 diabetes. Nat Genet 2007;39: 977-83.

22. Machiela MJ, Lindstrom S, Allen NE, et al. Association of type 2 diabetes susceptibility variants with advanced prostate cancer risk in the Breast and Prostate Cancer Cohort Consortium. Am J Epidemiol 2012;176:1121-9.

23. Waters KM, Wilkens LR, Monroe KR, et al. No association of type 2 diabetes risk variants and prostate cancer risk: the multiethnic cohort and PAGE. Cancer Epidemiol Biomarkers Prev 2011; 20:1979-81.

24. Ding EL, Song Y, Malik VS, et al. Sex differences of endogenous sex hormones and risk of type 2 diabetes: a systematic review and meta-analysis. JAMA 2006;295:1288-99.

25. Roddam AW, Allen NE, Appleby P, et al. Endogenous sex hormones and prostate cancer: a collaborative analysis of 18 prospective studies. $J$ Natl Cancer Inst 2008;100:170-83.

26. Robinson D, Garmo H, Bill-Axelson A, et al. Use of 5alpha-reductase inhibitors for lower urinary tract symptoms and risk of prostate cancer in Swedish men: nationwide, population based casecontrol study. BMJ 2013;346:f3406.

27. Giovannucci E, Michaud D. The role of obesity and related metabolic disturbances in cancers of the colon, prostate, and pancreas. Gastroenterology 2007;132:2208-25.

28. Roddam AW, Allen NE, Appleby P, et al. Insulin-like growth factors, their binding proteins, and prostate cancer risk: analysis of individual patient data from 12 prospective studies. Ann Intern Med 2008;149:461-71, W83-8.

29. Rajpathak SN, He M, Sun Q, et al. Insulin-like growth factor axis and risk of type 2 diabetes in women. Diabetes 2012;61:2248-54.

30. Turner EL, Lane JA, Donovan JL, et al. Association of diabetes mellitus with prostate cancer: nested case-control study (Prostate testing for cancer and treatment study). Int J Cancer 2011; 128:440-6.

31. Leitzmann MF, Ahn J, Albanes D, et al. Diabetes mellitus and prostate cancer risk in the Prostate,
Lung, Colorectal, and Ovarian Cancer Screening Trial. Cancer Causes Control 2008;19:1267-76.

32. Xu H, Jiang HW, Ding GX, et al. Diabetes mellitus and prostate cancer risk of different grade or stage: A systematic review and meta-analysis. Diabetes Res Clin Pract 2013;99:241-9.

33. Buzzai M, Jones RG, Amaravadi RK, et al. Systemic treatment with the antidiabetic drug metformin selectively impairs p53-deficient tumor cell growth. Cancer Res 2007;67:6745-52.

34. Stevens RJ, Ali R, Bankhead CR, et al. Cancer outcomes and all-cause mortality in adults allocated to metformin: systematic review and collaborative meta-analysis of randomised clinical trials. Diabetologia 2012;55:2593-603.

35. Zhang P, Li H, Tan X, et al. Association of metformin use with cancer incidence and mortality: a meta-analysis. Cancer Epidemiol 2013;37:207-18.

36. Patel SM, Iqbal N, Kaul S, et al. Effects of metformin and leuprolide acetate on insulin resistance and testosterone levels in nondiabetic postmenopausal women: a randomized, placebo-controlled trial. Fertil Steril 2010;94:2161-6.

37. Sarma AV, Burke JP, Jacobson DJ, et al. Associations between diabetes and clinical markers of benign prostatic hyperplasia among communitydwelling Black and White men. Diabetes Care 2008;31:476-82.

38. Sarma AV, Parsons JK, McVary K, et al. Diabetes and benign prostatic hyperplasia/lower urinary tract symptoms-what do we know? J Urol 2009; 182:S32-7.

39. Werny DM, Saraiya M, Gregg EW. Prostate-specific antigen values in diabetic and nondiabetic US men, 2001-2002. Am J Epidemiol 2006;164: 978-83.

40. Waters KM, Henderson BE, Stram DO, et al. Association of diabetes with prostate cancer risk in the multiethnic cohort. Am J Epidemiol 2009; 169:937-45.

41. Okura Y, Urban LH, Mahoney DW, et al. Agreement between self-report questionnaires and medical record data was substantial for diabetes, hypertension, myocardial infarction and stroke but not for heart failure. J Clin Epidemiol 2004; 57:1096-103.

42. Bansal D, Undela K, D'Cruz S, et al. Statin use and risk of prostate cancer: a meta-analysis of observational studies. PLoS One 2012;7: e46691.

43. Lehman DM, Lorenzo C, Hernandez J, et al. Statin use as a moderator of metformin effect on risk for prostate cancer among type 2 diabetic patients. Diabetes Care 2012;35:1002-7. 